\title{
Error Estimate of the Ares I Vehicle Longitudinal Aerodynamic Characteristics Based on Turbulent Navier-Stokes Analysis
}

\author{
Khaled S. Abdol-Hamid* and Farhad Ghaffari* \\ NASA Langley Research Center \\ Hampton, Virginia
}

Numerical predictions of the longitudinal aerodynamic characteristics for the Ares I class of vehicles, along with the associated error estimate derived from an iterative convergence grid refinement, are presented. Computational results are based on the unstructured grid, Reynolds-averaged Navier-Stokes flow solver USM3D, with an assumption that the flow is fully turbulent over the entire vehicle. This effort was designed to complement the prior computational activities conducted over the past five years in support of the Ares I Project with the emphasis on the vehicle's last design cycle designated as the A106 configuration. Due to a lack of flight data for this particular design's outer mold line, the initial vehicle's aerodynamic predictions and the associated error estimates were first assessed and validated against the available experimental data at representative wind tunnel flow conditions pertinent to the ascent phase of the trajectory without including any propulsion effects. Subsequently, the established procedures were then applied to obtain the longitudinal aerodynamic predictions at the selected flight flow conditions. Sample computed results and the correlations with the experimental measurements are presented. In addition, the present analysis includes the relevant data to highlight the balance between the prediction accuracy against the grid size and, thus, the corresponding computer resource requirements for the computations at both wind tunnel and flight flow conditions. NOTE: Some details have been removed from selected plots and figures in compliance with the sensitive but unclassified (SBU) restrictions. However, the content still conveys the merits of the technical approach and the relevant results.

Nomenclature

$\begin{array}{lll}C_{A F} & = & \text { axial force coefficient, } F_{X} / q_{\infty} S_{r e f} \\ C_{N} & = & \text { normal force coefficient, } F_{Z} / q_{\infty} S_{r e f} \\ C_{m} & = & \text { pitching moment coefficient, pitching moment } / q_{\infty} S_{r e f} X_{m r c} \\ C_{R M} & = & \text { rolling moment coefficient, rolling moment / } q_{\infty} S_{r e f} D \\ C_{Y} & = & \text { side force coefficient, } F_{Y} / q_{\infty} S_{r e f} \\ C_{Y M} & = & \text { yawing moment coefficient, yawing moment / } q_{\infty} S_{r e f} X_{m r c} \\ F_{X} & = & \text { axial force, lbf } \\ F_{Y} & = & \text { side force, lbf } \\ F_{Z} & = & \text { normal force, lbf } \\ \mathrm{hr} & = & \text { hour } \\ M & = & \text { Mach number } \\ N & = & \text { total number of cells; used for grid parameter estimation } \\ q_{\infty} & = & \text { free-stream dynamic pressure, psf } \\ R e & = & \text { Reynolds number based on D } \\ S_{r e f} & = & \text { reference area, ft } \\ X, Y, Z & = & \text { reference coordinate system } \\ X_{m r c} & = & \text { axial location of the moment reference center } \\ y^{+}{ }_{a v e} & = & \text { average inner law distance } \\ \alpha & = & \text { angle of attack, degrees } \\ \Delta & = & \text { incremental value } \\ \phi & = & \text { vehicle roll angle, also Phi, degrees }\end{array}$

Acronyms

ADAC $=$ Ares I design analysis cycle

* Aerospace Engineer, Configuration Aerodynamic Branch, Associate Fellow AIAA 


$\begin{array}{lll}\text { BDM } & = & \text { booster deceleration motor } \\ \text { BTM } & = & \text { booster tumble motor } \\ \text { BG } & = & \text { base grid } \\ \text { CEV } & = & \text { crew exploration vehicle } \\ \text { CFD } & = & \text { computational fluid dynamics } \\ \text { CP } & = & \text { center of pressure, } C_{m} / C_{N} \\ \text { CPU } & = & \text { central processing unit } \\ \text { DAC } & = & \text { design analysis cycle } \\ \text { D } & = & \text { diameter } \\ \text { FLT } & = & \text { flight } \\ \text { FS } & = & \text { first stage } \\ \text { LaRC } & = & \text { Langley Research Center } \\ \text { LAS } & = & \text { launch abort system } \\ \text { LH2 } & = & \text { liquid hydrogen feedline } \\ \text { NASA } & = & \text { National Aeronautics and Space Administration } \\ \text { IG } & = & \text { infinite-size grid } \\ \text { Max } & = & \text { maximum } \\ \text { PSWT } & = & \text { Polysonic Wind Tunnel } \\ \text { RCS } & = & \text { reaction control system } \\ \text { RoCS } & = & \text { roll control system } \\ \text { SA } & = & \text { Spalart-Allmaras } \\ \text { SRB } & = & \text { solid rocket booster } \\ \text { UPWT } & = & \text { Unitary Plan Wind Tunnel } \\ \text { US } & = & \text { upper stage } \\ \text { WT } & = & \text { wind tunnel }\end{array}$

\section{Introduction}

HE National Aeronautics and Space Administration's (NASA) Constellation Program [1] was initiated to address the needs of U.S. access to space in the post Space Shuttle era with mission to the Moon, Mars and beyond. Unlike the Space Shuttle, where both crew and cargo are launched simultaneously, the architecture for the Constellation Program proposed two separate launch vehicles: the Ares I for the crew and the Ares V for the cargo. The Ares I, the vehicle of interest in the present study, is a two-stage rocket that incorporates a modified five-segmented solid rocket booster (SRB) from the Shuttle program while the upper stage is powered by the redesigned J-2X engine from the Apollo Program. Figure 1 shows, conceptually, the major components of the vehicle as well as the proposed nominal flight trajectory. The Ares I major components consist of the launch abort system (LAS), crew exploration vehicle (CEV) named Orion, the upper stage (US), and the first stage (FS) SRB that includes the frustum and the aft skirt. 


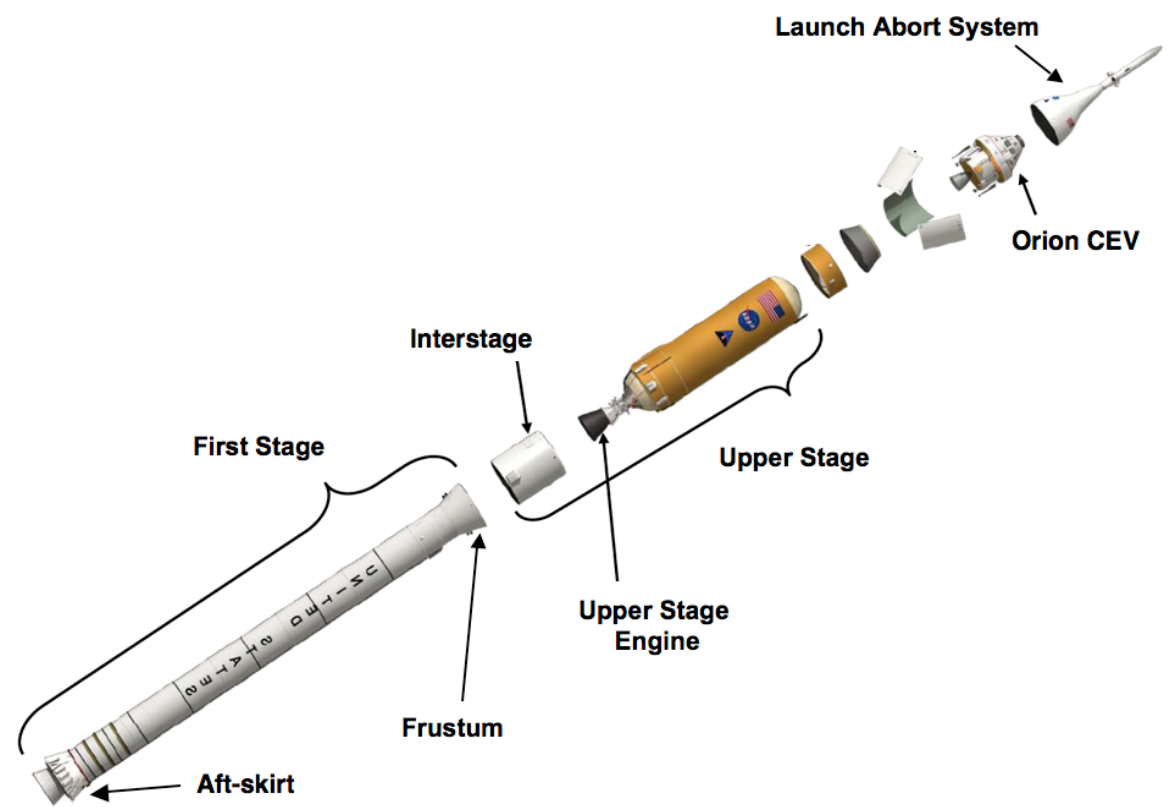

a) Major components.

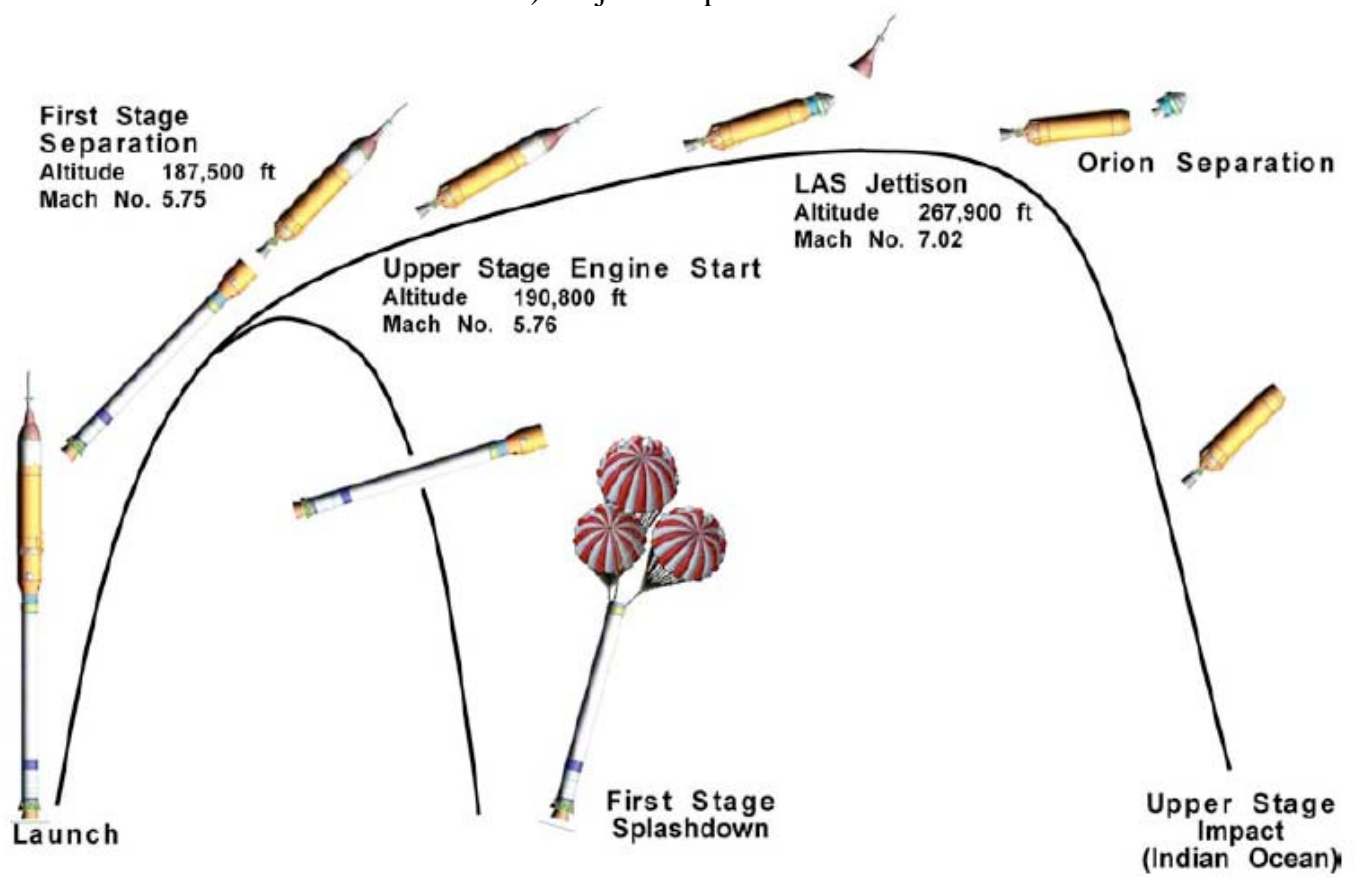

b) Nominal flight trajectory [2].

Figure 1. The Ares I major components and its nominal flight trajectory.

Complementary to ground based wind tunnel testing and flight tests, computational fluid dynamic (CFD) methods are being extensively used to aid the design and the ascent aerodynamic data development of the proposed crew launch vehicle. One such CFD method is the NASA Langley Research Center (LaRC) developed Navier-Stokes flow solver USM3D [3] that was designated to have a primary role within the Ares project to develop the necessary computational aerodynamic data while other widely used flow solvers, FUN3D [4] and OVERFLOW [5], were to have supporting roles to provide complementary results for fewer cases as part of a confidence building process to ensure code-to-code solution consistency. The free-stream flow conditions considered typical for the nominal ascent flight trajectory generally ranged over $0^{\circ}<\alpha<8^{\circ}, 0.5<M<4.5$, at 
various vehicle roll angles $(\phi)$ over 360 degrees.

A typical Ares I vehicle contains numerous protuberances, adding to the complexity of the geometry and the resulting flow physics both from the numerical simulation aspect as well as the wind tunnel model design, manufacturing, and testing. For example, Fig. 2 shows a translucent front view of such a vehicle. The figure also shows a list of the individual and the groups of protuberances along with the abbreviations used in the analysis. Such protuberances include the LAS nozzles, CEV umbilical cover, many others over the US and the FS such as the roll control system (RoCS), reaction control system (RCS), liquid hydrogen (LH2) feedline, systems tunnel, booster deceleration motors (BDMs), booster tumbling motors (BTMs), hold-down wedges, and many other smaller ones such as cameras, antennas, and rings.

2 upper stage reaction control systems ( 2 US RCS)

2 upper stage roll control systems (2 RoCS)

1 umbilical cover (1 Umbil)

4 upper stage ullage motors (4 US Ullage)

1 first stage tunnel (1 FSTUN)

1 upper stage tunnel (1 USTTUN)

2 booster tumbling motors (2 frustum BTM)

2 upper stage cameras ( 2 US CAM)

1 launch abort system tunnel (1 LAS tunnel)

2 upper stage ultra high frequency antennae (2 US UHF)

8 booster deceleration motors (8 BDMs)

1 upper stage liquid hydrogen feedline ((1 US LH2)

4 launch abort system nozzles (4 LAS Nozzles)

1 upper stage fill/drain-line (1 US F/D-line)

4 wedges

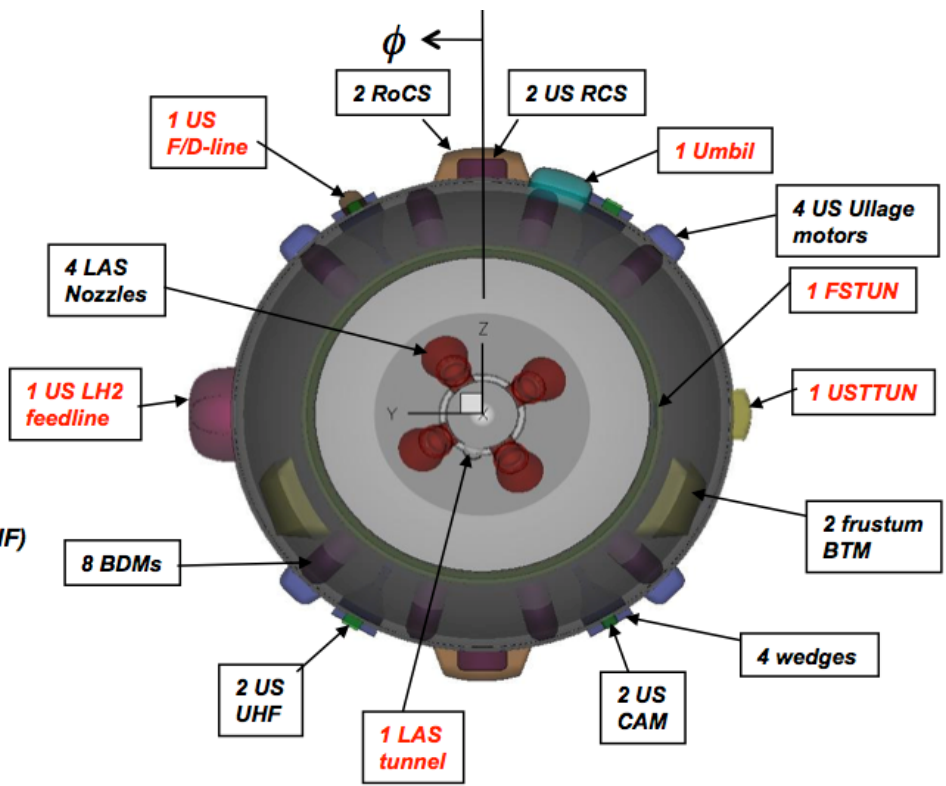

Figure 2. Typical Ares I vehicle with protuberances.

An overview of the prior computational results and analyses along with the processes used to assess, verify, and validate the numerical aerodynamic predictions is presented in reference [6] for the evolving Ares I design analysis cycles (ADAC). As discussed in the reference, the USM3D results were verified against those obtained from other Navier-Stokes flow solvers, whereas the validity was assessed through comparisons with the available wind tunnel data. In spite of many efforts made during the prior computations to address the solution sensitivities to the grid refinement as part of the solution verification process, a systematic approach was undertaken in the present study to tackle the issue by providing the estimates of the computational error in the predicted longitudinal aerodynamic coefficients of the ADAC-3 (A106) configuration with full protuberances. The computations were performed at the nominal ascent flight trajectory and without including any propulsion effects. The initial analyses were conducted at wind tunnel flow conditions for which the appropriate experimental data are available to gauge the validity of the numerical predictions and the associated error estimates. Once established, the procedures were then applied to compute the aerodynamic estimates at representative flight Reynolds number flow conditions for which no experimental data existed. In addition, the present analysis includes the relevant data to distinguish the balance between the prediction accuracy against the grid size and the corresponding computer resource requirements for the computations at both wind tunnel and flight flow conditions. Due to the limited availability of the computer resources, the present analyses were performed only at the selected flow conditions of $\alpha=8^{\circ}, M=0.9,1.6,3.0$ from those used in the prior computations [6]. The detail analysis presented in this paper only focuses on the numerical data obtained at $\alpha=$ $8^{\circ}, M=1.6$ flow conditions, which is considered to be near the maximum dynamic pressure that the vehicle would experience during the ascent trajectory. However, a summary of the final results that encompasses the entire computational matrix is presented for completeness. 


\section{Computational Approach}

\section{USM3D Flow Solver}

USM3D is a tetrahedral cell-centered, finite volume, Euler and Navier-Stokes flow solver. Time integration follows the implicit point Gauss-Seidel algorithm, explicit Runge-Kutta approach, and local time stepping for convergence acceleration. The solver scheme allows various options for computing the inviscid flux quantities across each cell face. These include Roe's flux-difference splitting (FDS), advection upstream splitting method (AUSM), flux vector splitting (FVS), and the Harten, Lax, and van Leer with contact restoration (HLLC) scheme. MinMod and Superbee flux limiters have been incorporated in the flow solver to smooth out the flow discontinuities due to shock waves. Turbulence models include Spalart-Allmaras (SA) [7] with and without the wall function, $k-\varepsilon$ [8], shear stress transport (SST) [9], and algebraic stress models. In addition to the standard slip/no-flow through (inviscid surface) and the no-slip/no-flow through (viscous surface) boundary conditions, the method also includes a transpiration boundary condition that can be used to simulate propulsion effects. Although USM3D is used mainly for ideal gas simulation with constant gamma, options for variable gamma and limited real gas effects are also included in the solver.

\section{Grid Generation}

The surface triangulations along with the field tetrahedral volume grids were generated using the GRIDTOOL [10] and VGRID [11] software developed at LaRC. A rectangular box that encompasses the vehicle is typically used to define the computational domain far-field boundaries. Each face of this rectangular box is located approximately several body lengths away from the configuration in the upstream, radial and downstream direction. All computational grids used in the present analysis included a sting geometry representation of the WT model. The detail grid description used for the computations at WT Re and FLT Re will be discussed later. As a general practice, the analysis of each final converged solution is conducted to insure that the average turbulent boundary sub-layer, $y^{+}{ }_{a v e}$, has been grid resolved to equal to or less than 1 .

\section{Solution development and convergence}

Many efforts were made in the prior activities to establish the appropriate parameter settings for the USM3D flow solver, such as the flux schemes, limiters, and turbulence model to ensure the solution development remained stable over the nominal ascent range of the flow conditions [6]. This led to the development of an efficient script that would automatically generate appropriate input-stream parameters to the flow solver and submit a series of cases such as a Mach number or angle of attack sweep to a designated computer platform. Upon completion, a summary page pertinent to the solution convergence characteristics would be generated for the validity assessments. Figure 3 shows a typical summary page for the ADAC-3 (A106) configuration. This summary page includes the designed statistical convergence data for the longitudinal aerodynamic and rolling moment coefficients as well as the flow solution residuals.
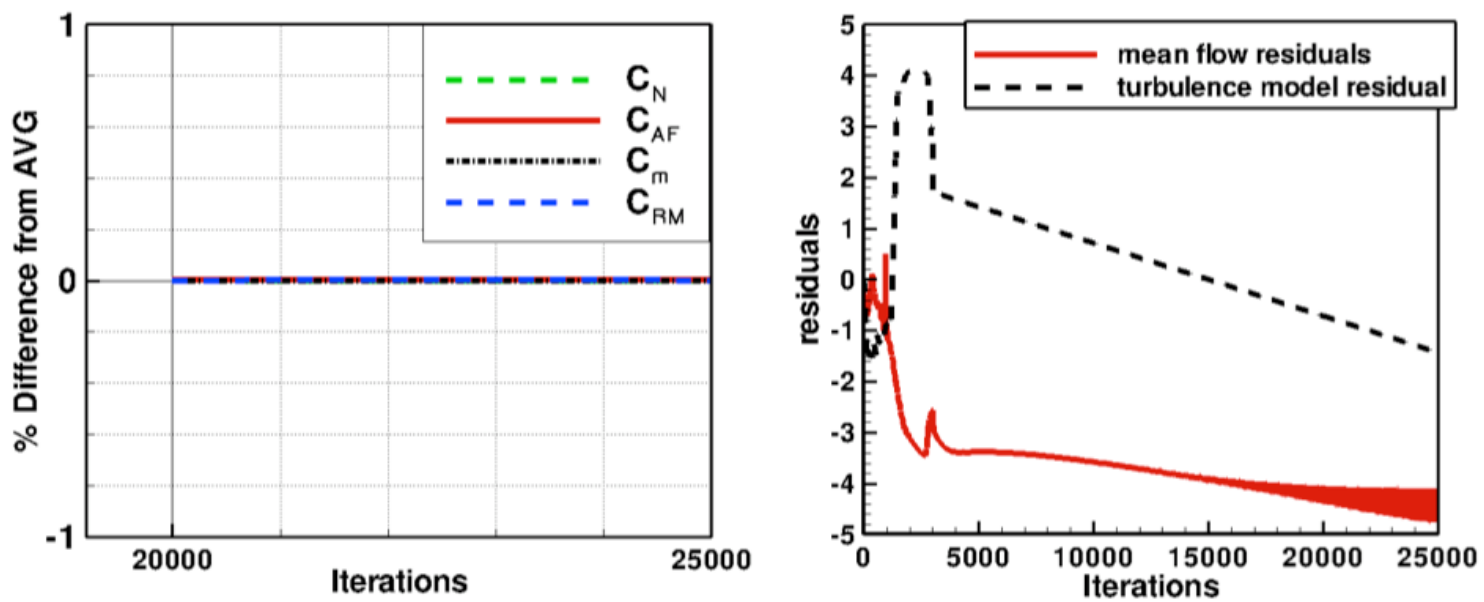

Figure 3. Typical solution convergence for the ADAC-3 (A106), $M=1.6, \alpha=8^{\circ}, \phi=0^{\circ}$, WT Re.

Solution convergence was evaluated by monitoring the overall fluctuations in all six force and moment coefficients. In particular, a solution is considered iteratively converged when the fluctuations in $C_{N}, C_{A F}, C_{m}$, and $C_{R M}$ become generally less than $0.5 \%$ of its average final value over the last 2000 iterations (denoted as 
AVG in the plots of Fig. 3) and about a 2-5 orders of magnitude drop in the mean flow residuals and turbulent model residual. As a result, the error estimate due to iterative solution convergence is considered very small and therefore is not discussed any further in the present analysis. All present computations are based on the SA turbulence model and have been performed on the Pleiades computer system at the NASA Advanced Supercomputer (NAS) complex located at the Ames Research Center.

\section{Results and Discussions}

\section{Computations at wind tunnel Re}

The guidelines established by the Drag Prediction Workshop [12] and the High Lift Prediction Workshop [13] to address solution sensitivity to grid refinement was adopted for estimating the computational error in the previously computed longitudinal aerodynamic coefficients [6]. The results obtained from the base grid (BG) in the prior computations provided the reference data for the present analysis and, in particular, for generating the properly sized coarse and fine grid levels. Figure 4 shows the representative computational grids on the surface and the near-field view of a typical cross-sectional cut for the coarse, base, and fine grid. There were roughly over 40 protuberances, with different sizes and shapes, which were distributed nonuniformly over the configuration. Such geometrical complexity presented a challenge for the grid generation and the flow solver to properly capture the associated flow physics and the resulting aerodynamic properties of the vehicle across a fairly wide range of flow conditions. Table 1 shows sample results for the number of surface triangular elements for the selected major protuberances and their corresponding percent ratio to that of the total vehicle. Approximately $35 \%$ of the total number of surface elements for the vehicle was used to represent these major protuberances.

Relative to the base grid (BG), a factor of three was used to size the corresponding number of cells in the coarse and fine grid as shown in Table 2. Based on this approach, the coarse and the fine grid consisted of onethird and three times the number of cells contained in the BG, respectively. These ratios were also maintained approximately the same in both the viscous boundary layer region as well as in the inviscid field. Furthermore, a factor of 1.26 was used as the grid growth rate $\left(\delta_{\mathrm{j}}\right)$ in the radial direction based on the following equation from Ref. 11 by setting the parameters $R 1=0.16$ and $R 2=0.04$ for all the grids used in the present investigation.

$$
\delta_{j}=\delta_{1}\left(1+R 1(1+R 2)^{j-1}\right)^{j-1}
$$

In this equation, the variable $\delta_{1}$, is the first cell height next to the solid surface, in the normal direction.
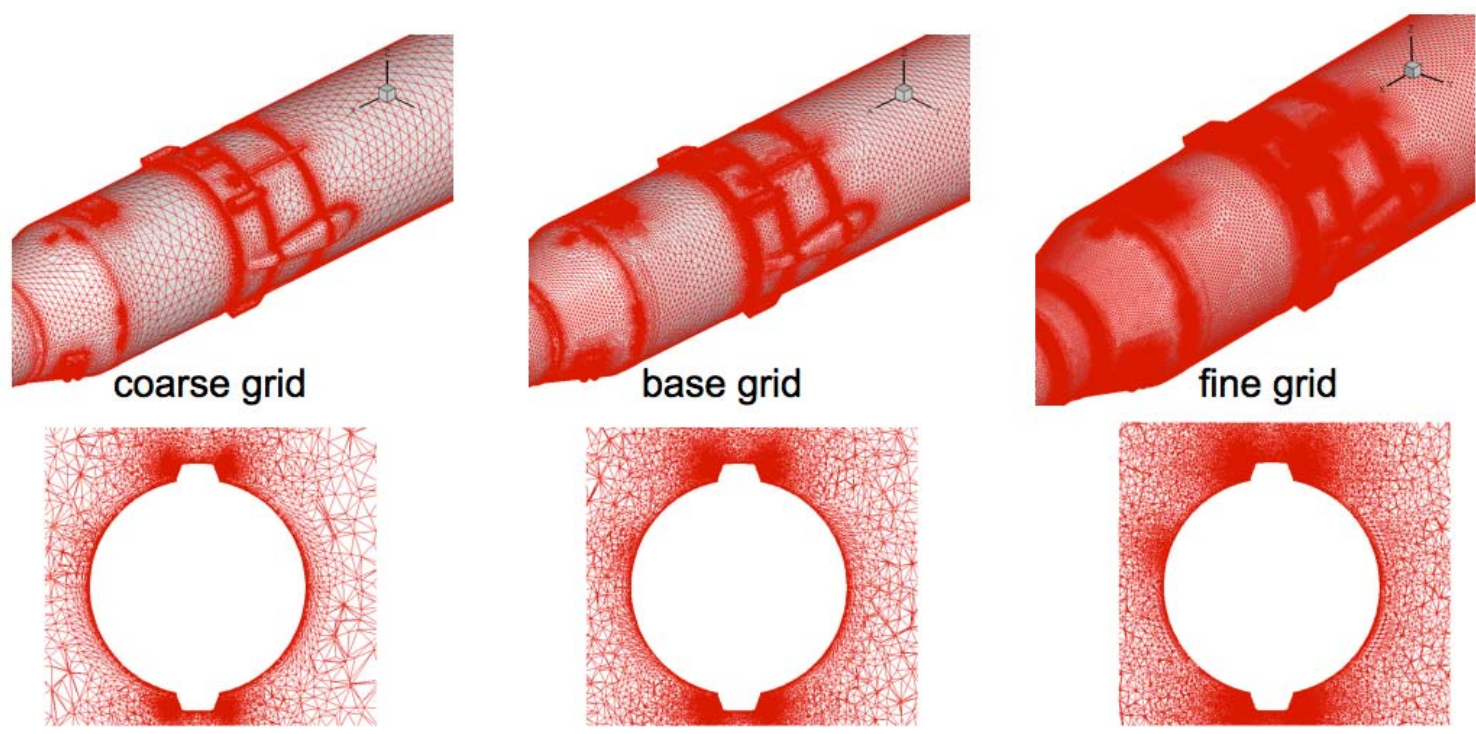

Figure 4. Computational grid distribution on the surface and a typical cross-flow plane for the coarse, base, and fine grids for the ADAC-3 (A106) configuration. 
Table 1 Sample surface grid elements for the major protuberances: base grid

\begin{tabular}{|c|c|c|}
\hline Protuberance & $\begin{array}{c}\text { Total number of surface } \\
\text { elements }\end{array}$ & $\begin{array}{c}\text { \% Ratio to the Total Surface } \\
\text { Elements }\end{array}$ \\
\hline 10 BDMs & 63,166 & 6.2 \\
\hline 2 BTMs & 60,544 & 5.9 \\
\hline First Stage Tunnel & 17,517 & 1.7 \\
\hline 4 LAS Nozzles & 27,677 & 2.7 \\
\hline LH2 & 18,575 & 1.8 \\
\hline Pressure Tunnel & 17,265 & 1.7 \\
\hline 2 RCS & 23,340 & 2.3 \\
\hline 2 RoCS & 58,680 & 5.7 \\
\hline 4 Ullage Motors & 50,760 & 5.0 \\
\hline Upper Stage Tunnel & 22,536 & 2.2 \\
\hline
\end{tabular}

The computational domain far-field boundaries were chosen to be at about 100 SRB diameters away from the vehicle. Table 2 also provides the values of $\delta_{1}$, the total number of cells in the viscous layer and the total number of surface elements for different grids. In addition, Table 2 presents the approximate range for the number of iterations along with the computer resource requirements (i.e., CPU-hr range) for a typical case. In an effort to improve the grid quality, a grid optimizer [14] was employed to smooth out any local grid skewness or a large variation of the cell volumes prior to the launching of the flow solver. This activity was shown to stabilize and enhance the solution convergence characteristics over the examined range of flow conditions. In addition, as shown in Table 2, there was roughly the expected linear relationship between the total number cells and the corresponding computer resource requirements. As a result, the fine grid computations required approximately three times the CPU hours to converge the solution as those needed for the BG. This similarity in computer usage remained roughly the same between the base and the coarse grid.

Table 2 ADAC-3 (A106) grid statistics and computer resource requirements for WT Re cases

\begin{tabular}{|c|c|c|c|c|c|c|}
\hline Grid & $\begin{array}{c}\text { Number of } \\
\text { cells, } \boldsymbol{N}\end{array}$ & $\mathbf{1}^{\text {st }}$ cell height, \\
$\boldsymbol{\delta}_{\mathbf{1}}$, inches & $\begin{array}{c}\text { Number of } \\
\text { viscous cells }\end{array}$ & $\begin{array}{c}\text { Number of } \\
\text { surface } \\
\text { elements }\end{array}$ & \#Iterations & $\begin{array}{c}\text { Computer } \\
\text { Resources } \\
\text { CPU-hr range }\end{array}$ \\
\hline coarse & $22,502,721$ & 0.0225 & $17,713,428$ & 377,272 & $15,000-25,000$ & $600-1000$ \\
\hline base & $70,203,102$ & 0.015 & $46,827,963$ & $1,023,590$ & $15,000-25,000$ & $2,000-4,000$ \\
\hline fine & $214,373,376$ & 0.01 & $115,818,453$ & $2,568,446$ & $15,000-25,000$ & $6,000-10,000$ \\
\hline
\end{tabular}

The coarse, base, and the fine grids were used to compute the flow at $\alpha=8^{\circ}, M=0.9,1.6,3.0$, and appropriate wind tunnel $R e$. These computations were performed at nominal roll angles $(\phi)$ ranging from $0^{\circ}$ to $360^{\circ}$ at $30^{\circ}$ increment. Figure 5 shows the effects of the grid refinement on the computed $C_{A F}$ and $C_{N}$ at the representative flow conditions of $\alpha=8^{\circ}$ and $M=1.6$ as a function of grid parameter $\left(N^{-2 / 3}\right)$ for various roll angles. The results for both $C_{A F}$ and $C_{N}$ show a nearly second-order asymptotic behavior approaching a limiting value for the infinite-size grid (IG) over the vertical axis. It should be noted that the grid parameter increment $\left(\Delta N^{-2 / 3}\right)$ between the base and the fine grid in roughly the same as that of the fine and the IG. This feature was indicative of the appropriateness on the total number of cell size selections between the base and the fine grid that resulted in roughly a linear extrapolation to obtain the IG values. Similarly, Fig. 6 shows the procedure used for extrapolating the computed $C_{A F}$ at various roll angles to determine the limiting value for an IG. The top 
portion of the figure shows the computed $C_{A F}$ for various roll angles and the extrapolation step for two selected roll angles of $30^{\circ}$ and $240^{\circ}$. The lower left portion of the figure shows the limiting values of the coefficient, determined from an extrapolation of the results from the BG to an IG by an error bar-lines for all computed roll angles. The lower right portion of the figure shows the percent variation of the computed $C_{A F}$ for the limiting values from the BG to be less than about $4 \%$ for all the roll angles and that the maximum occurs at $\phi=30^{\circ}$.

A similar procedure was employed to determine the limiting values of the computed $C_{N}$ for various roll angles with the results shown in Fig. 7. Analogous to the $C_{A F}$, the computed $C_{N}$ also showed a monotonic behavior with grid refinement. The percent change of the $C_{N}$ limiting values were shown to be less than $10 \%$ for all the roll angles and that the maximum occurred at $\phi=300^{\circ}$.
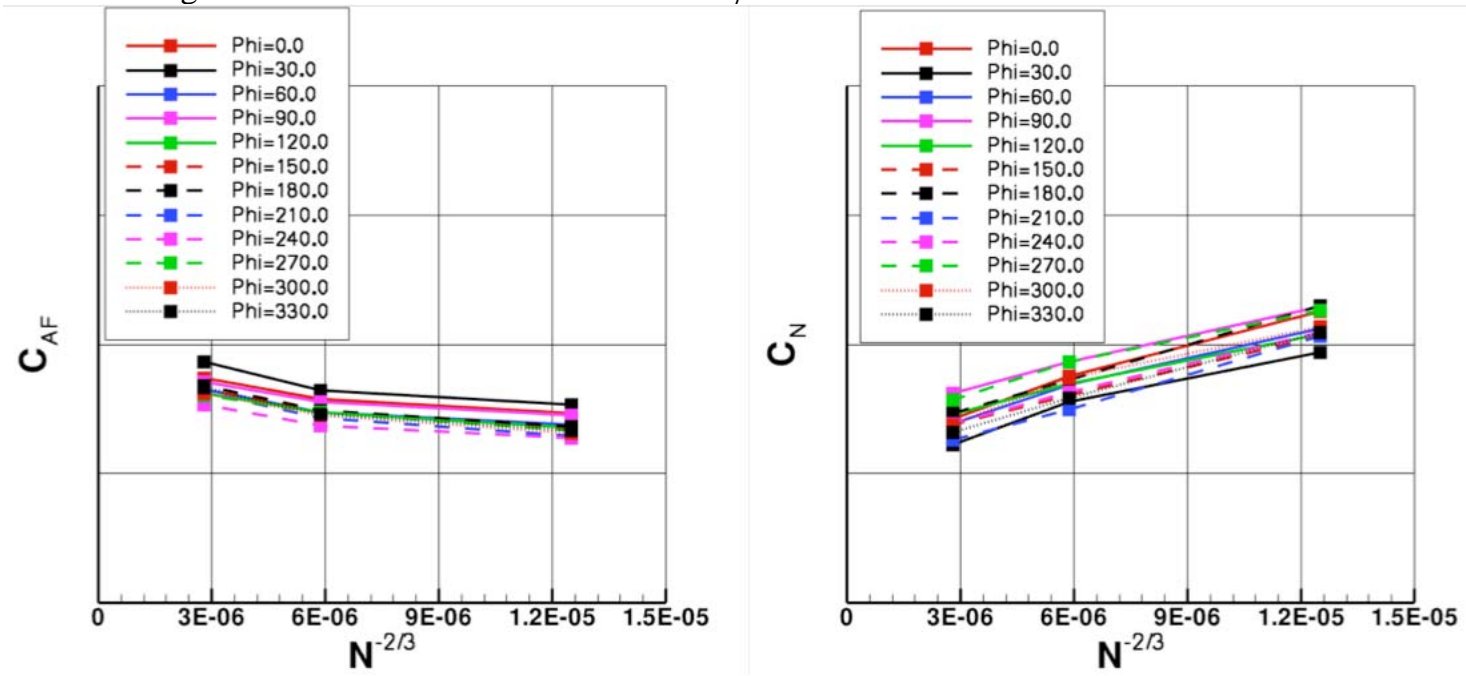

Figure 5. Effects of grid refinement on computed $C_{A F}$ and $C_{N}$ at various roll angles, $M=1.6, \alpha=$ $8^{0}$, WT Re.
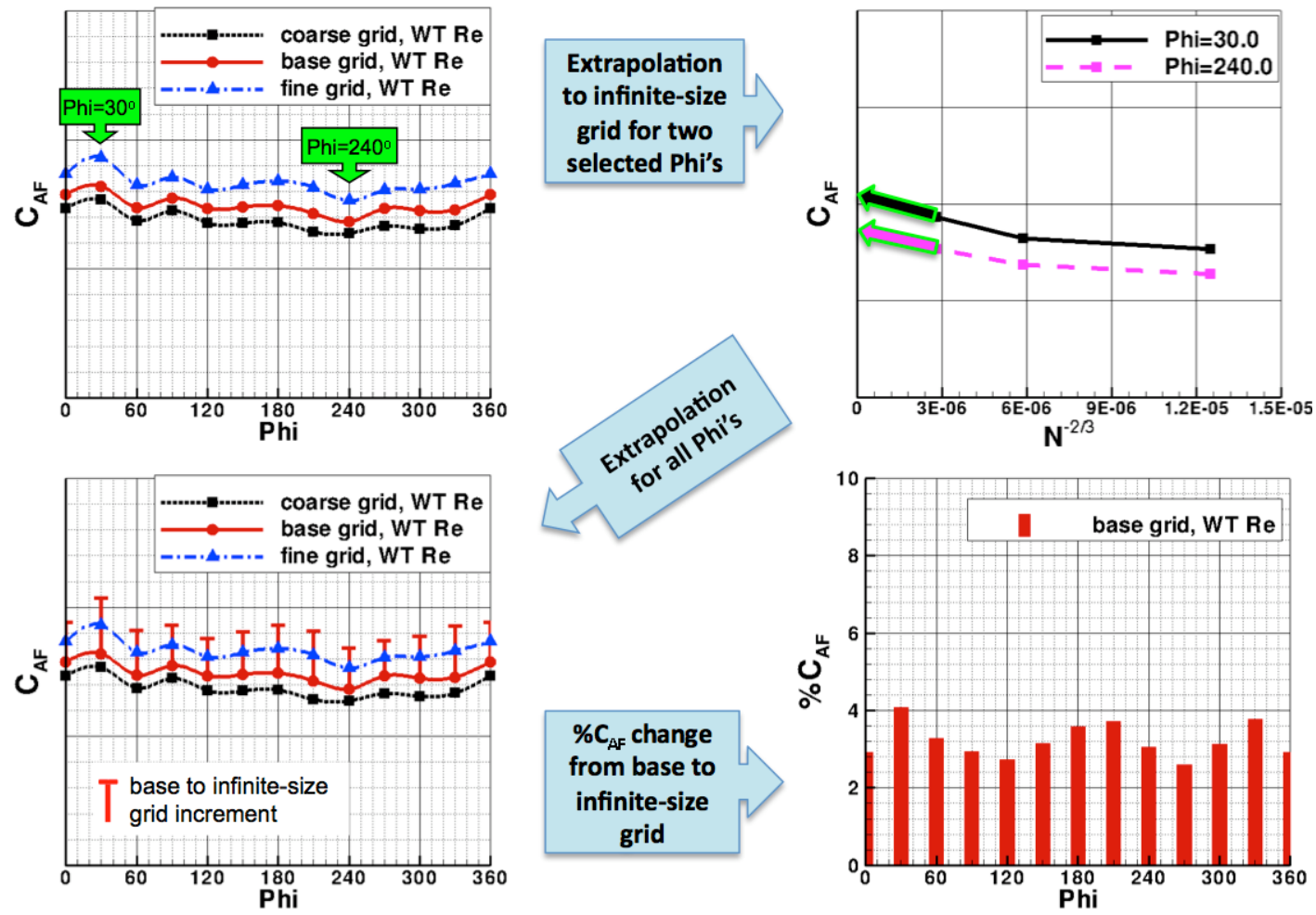

Figure 6. Extrapolation procedure to determine the $C_{A F}$ for an infinite-size grid and the percent error estimates from the base grid results, $M=1.6, \alpha=8^{0}$, WT $R e$.

8

American Institute of Aeronautics and Astronautics 

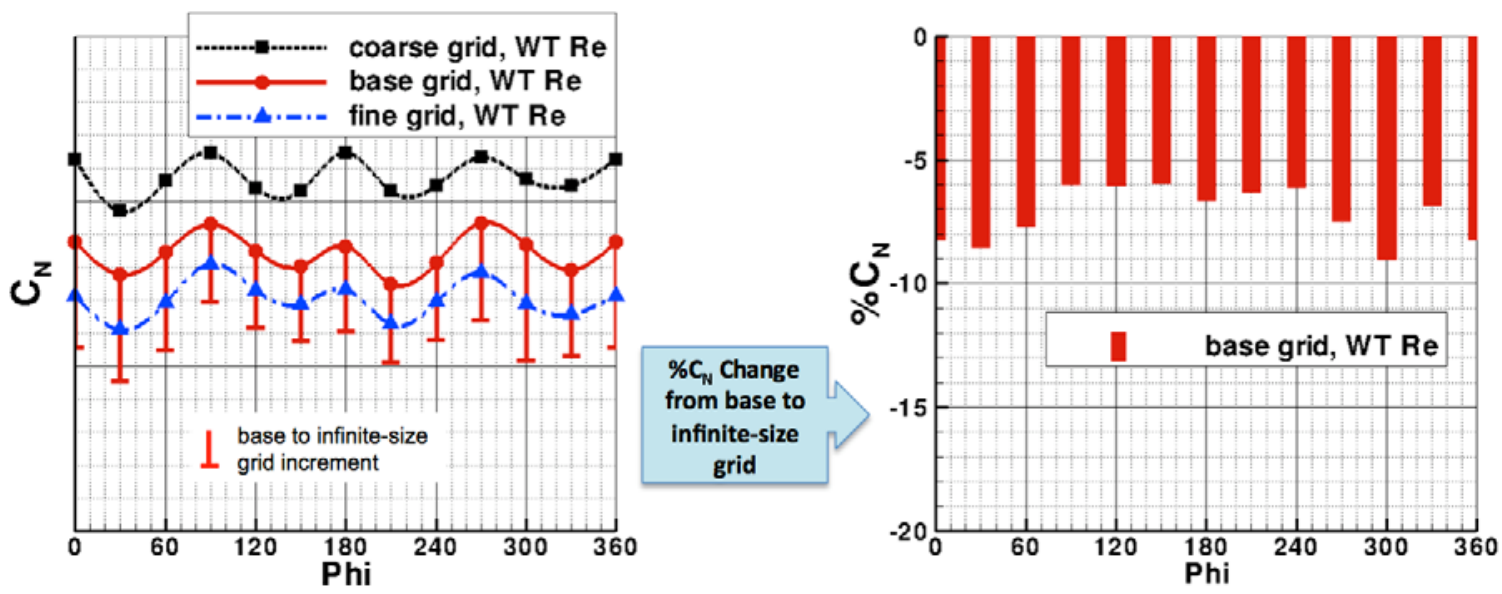

Figure 7. Effects of grid refinement on computed $C_{N}$ and the corresponding percent error estimates from base grid to an infinite-size grid, $M=1.6, \alpha=8^{\circ}$, WT Re.

Figure 8 shows the effects of iterative convergence grid refinement on the computed longitudinal aerodynamic coefficients as well as the center of pressure for the examined Mach numbers at WT Re. These data were the maximum error bar-lines or the limiting values of the extrapolated results from the base to the infinite-size grid across the entire range of roll angle. The results generally indicated a less than $10 \%$ variation in the computed coefficients due to the grid refinement over the examined flow conditions. In addition, the results also showed that the largest grid dependency occurred at transonic and low supersonic flow conditions for the computed $C_{N}$ and $C_{m}$ and that the overall variations decreased dramatically to less than $3 \%$ at the higher Mach number of 3.

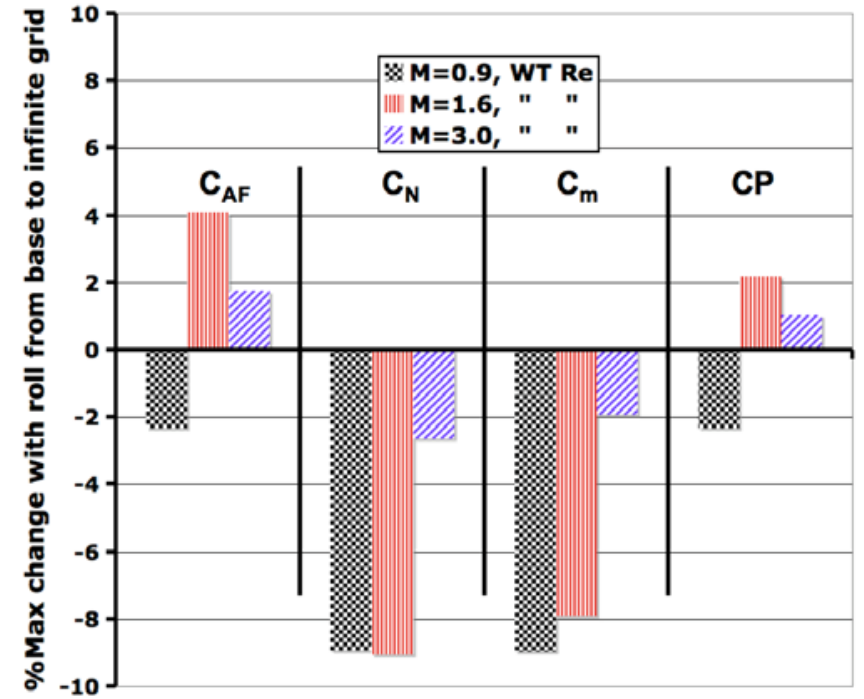

Figure 8. Percent maximum error estimate with roll angle in the computed longitudinal aerodynamic characteristics and the center of pressure from the base to an infinite-size grid for various Mach numbers at $\alpha=8^{\circ}$, WT Re.

The computed $C_{A F}$ results from the base grid and the extrapolated values for the infinite-size grid (shown with the error bar-lines) were correlated with the available experimental data in Fig. 9 for various roll angles at $M=1.6, \alpha=8^{\circ}$. The experimental data were obtained from the LaRC Unitary Plan Wind Tunnel (UPWT) [15] and the Boeing Polysonic Wind Tunnel (PSWT) [16]. These experimental results, developed within the Ares I project, are provided here without including any estimates of the errors associated with the measured data. The 
results, shown on the left side of the figure, indicate that the BG clearly underpredict the measured $C_{A F}$ across the roll angles; however, an improved correlation has been achieved between the IG and the wind tunnel data. As shown on the right side of the figure, the percent deviation between the computed $C_{A F}$ from the BG and the average values between the two wind tunnel data were less that $5 \%$ whereas it was less than $1 \%$ for the IG across the examined roll angles.
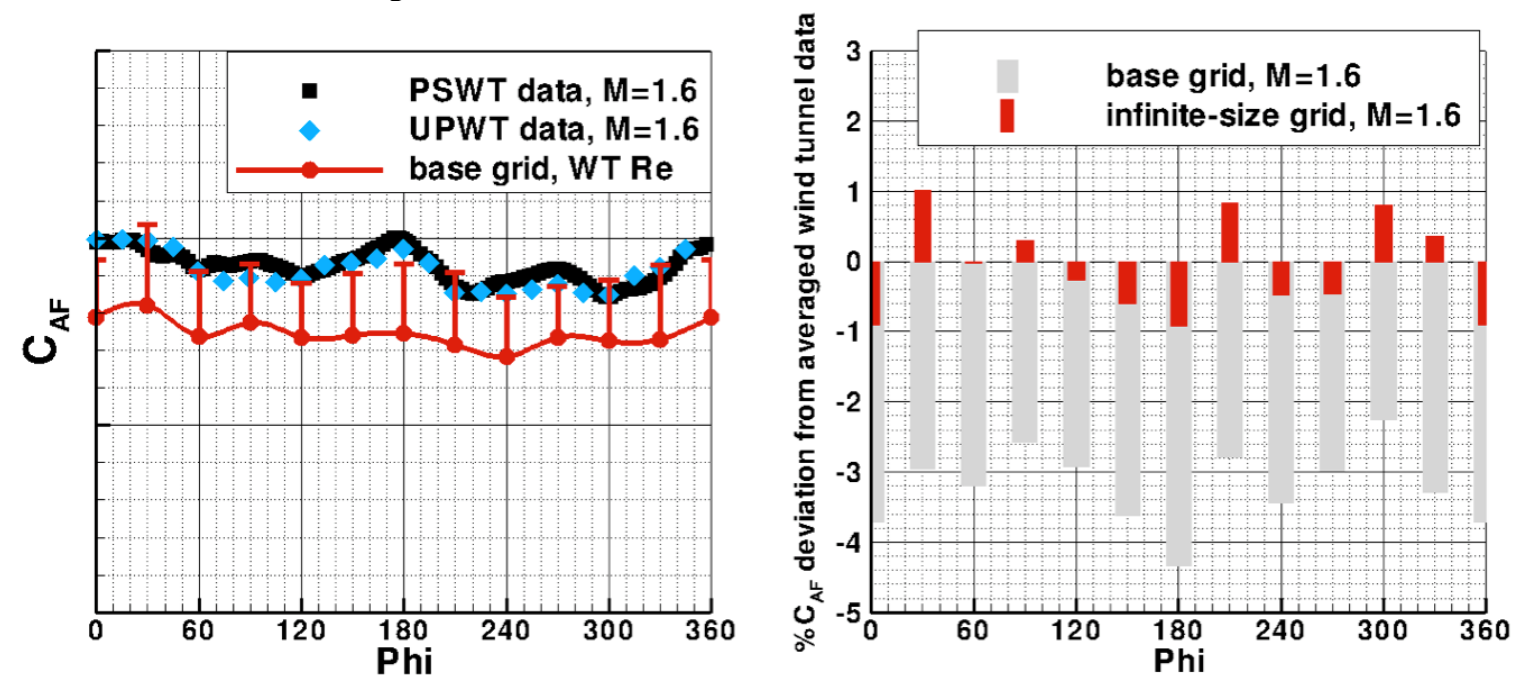

Figure 9. Computed $C_{A F}$ from the base and infinite-size grid and correlation with wind-tunnel data along with their percent deviation, $M=1.6, \alpha=8^{\circ}$.

Similarly, the computed $C_{N}$ results obtained from the BG and the extrapolated values from the IG were correlated with the measured wind tunnel data in Fig. 10 for various roll angles. The results, shown on the left side, indicate an overprediction of the computed $C_{N}$ using the BG at all examined roll angles, while an improved correlation has been achieved between the IG predictions and the average values of measured data from the two wind tunnels. As shown on the right side of Fig. 10, such an improved correlation was within a 5\% deviation as opposed to less than an $8 \%$ deviation for the computed results with the BG from the averaged measured data. Although not shown here, it should be noted that such an improved correlation in the computed $C_{A F}$ and $C_{N}$, between the IG and experimental data, was not relatively as well behaved for the pitching moment coefficient at a few roll angles despite the presence of a monotonic trend. However, the percent deviation of the computed $C_{m}$, using the BG and the IG, from the averaged wind tunnel data still remained well within $8 \%$ across the examined roll angles.
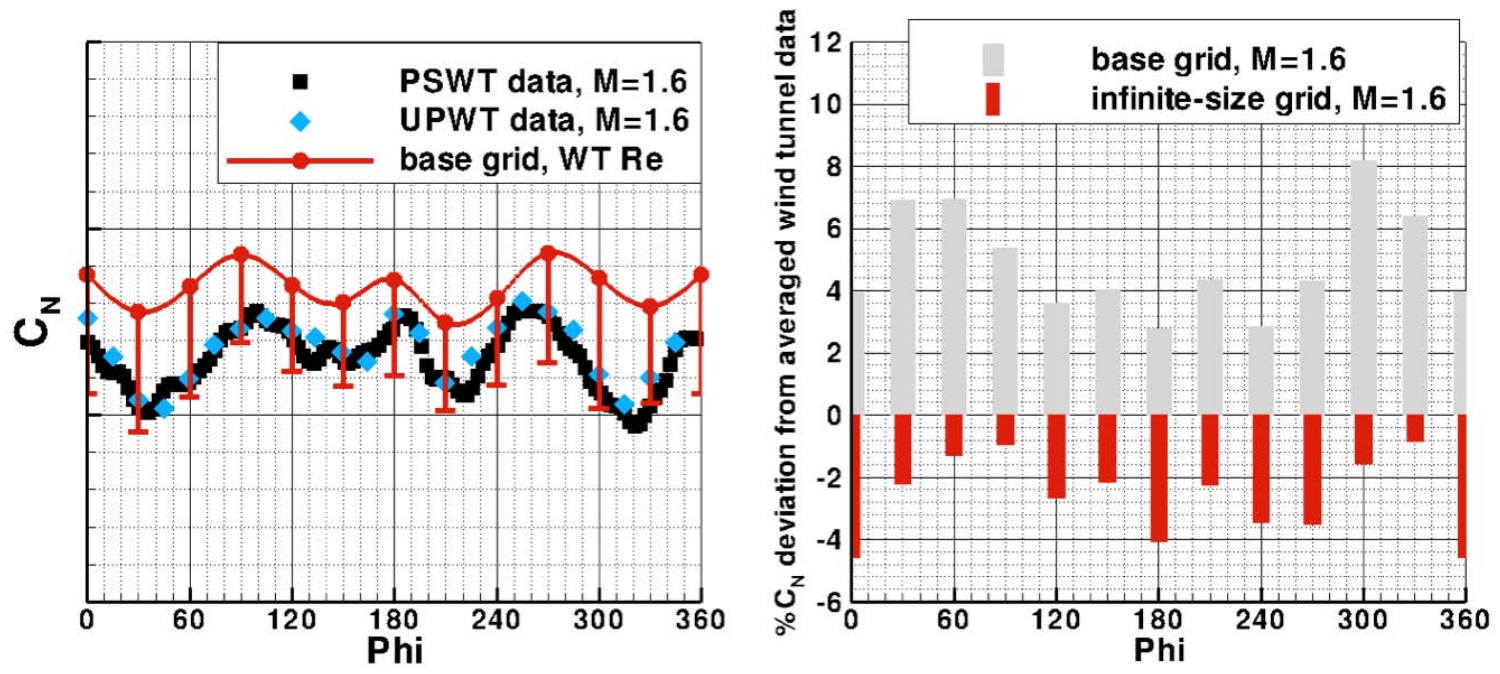

Figure 10. Computed $C_{N}$ from the base and infinite-size grid and correlation with wind tunnel data along with their percent deviation, $M=1.6, \alpha=8^{\circ}$.

10

American Institute of Aeronautics and Astronautics 
Figure 11 presents the maximum deviation in the computed longitudinal aerodynamic coefficients from the WT data, across the examined roll angles using both the BG and IG, for various Mach numbers. The results based on the BG at the transonic Mach number indicated the highest deviation of roughly $23 \%$ associated with the $C_{N}$ but improved to approximately $16 \%$ for the computations with the IG. However, the results at the supersonic flow conditions based on the BG showed a maximum deviation of only about $8 \%$ for all the computed coefficients with that level improving to approximately 5\% for the IG.

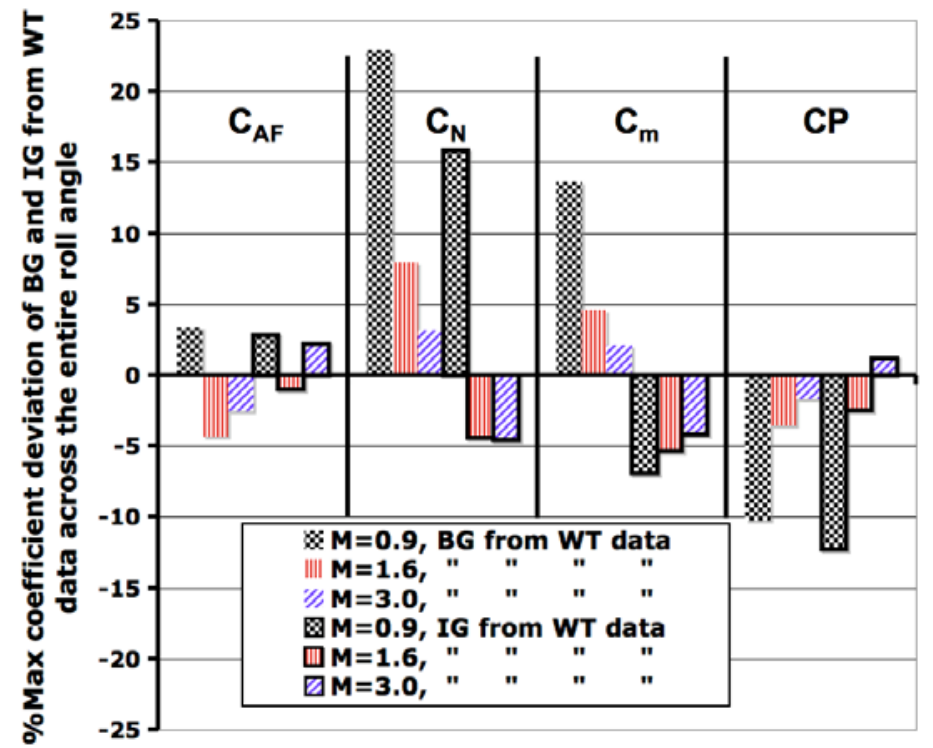

Figure 11. Maximum deviations in the computed longitudinal aerodynamic coefficients from the WT data across the examined roll angles using the BG and IG for various Mach numbers.

\section{Computations at Flight Re}

Same surface triangle definitions, used to generate the field grids for the WT $R e$ calculations, were employed to generate the corresponding volume grids for the flight Reynolds number computations. Table 3 shows the grid statistics and the computer resource requirements for FLT Re computations. Finer grid spacing was used primarily in the viscous layer to resolve the expected thinner boundary layer flow characteristic that was at least an order of magnitude higher $R e$ than WT flow conditions. Such an effect caused an increase of about $20 \%$ to $30 \%$ more volume cells for the FLT Re cases than their WT Re counterpart grids. As expected, such an increase in the total number of cells resulted in the additional computer resource requirements for the computations at FLT Re.

Table 3 ADAC-3 (A106) grid statistics and computer resource requirements for FLT Re cases

\begin{tabular}{|c|c|c|c|c|c|c|}
\hline Grid & $\begin{array}{c}\text { Number of } \\
\text { cells, } \boldsymbol{N}\end{array}$ & $\begin{array}{c}\mathbf{1}^{\text {st }} \text { cell height, } \\
\boldsymbol{\delta}_{\mathbf{1}} \text {, inches }\end{array}$ & $\begin{array}{c}\text { Number of } \\
\text { viscous cells }\end{array}$ & $\begin{array}{c}\text { Number of } \\
\text { surface } \\
\text { elements }\end{array}$ & \#Iterations & $\begin{array}{c}\text { Computer } \\
\text { Resources } \\
\text { CPU-hr range }\end{array}$ \\
\hline coarse & $29,809,726$ & 0.0015 & $24,741,678$ & 377,272 & $15,000-25,000$ & $800-1,200$ \\
\hline base & $90,922,136$ & 0.001 & $66,390,036$ & $1,023,590$ & $15,000-25,000$ & $3,000-4,000$ \\
\hline fine & $267,733,262$ & 0.00066 & $165,583,035$ & $2,568,446$ & $15,000-25,000$ & $10,000-15,000$ \\
\hline
\end{tabular}

Complementary computations at FLT Re were performed at the selected flow conditions and the roll angles. Figure 12 shows the incremental effects in the computed $C_{A F}$ and $C_{N}$, due to $\operatorname{Re}$ obtained from the BG and the IG for $M=1.6$ and $\alpha=8^{\circ}$. Two general observations can be made from these results at these particular flow conditions. The first is that the computed coefficients at FLT Re were both lower than those obtained at 
WT Re across all the roll angles. The second observation is that, surprisingly, the Re incremental effects were, for the most part, smaller than the ones computed because of the grid refinements. As a result, it can be concluded that the numerical predictions at the WT Re tend to provide more conservative estimates of these aerodynamic coefficients from a structural design standpoint for the vehicle than their counterparts at flight flow conditions. Therefore, these results suggest that future numerical activities should mainly focus on the computations at WT Re with only limited and strategically chosen cases to address the incremental effects due to WT to FLT Reynolds numbers.
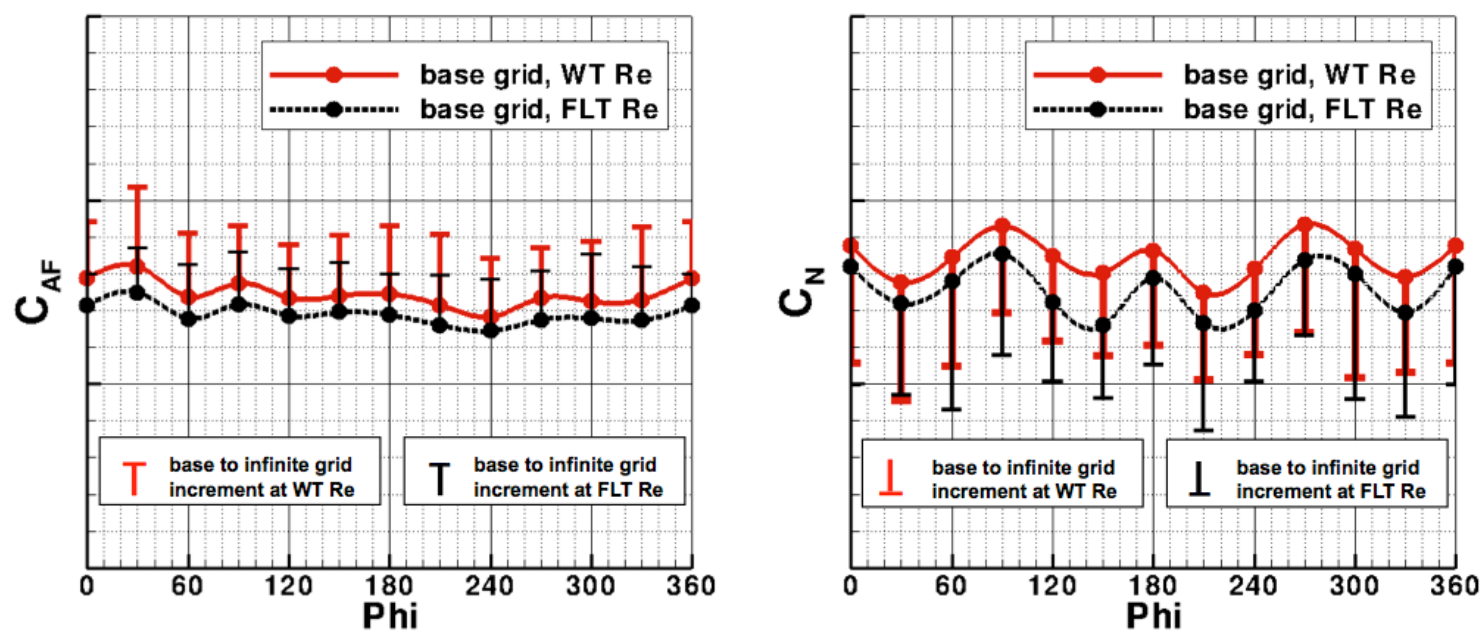

Figure 12. Computed $C_{A F}$ and $C_{N}$ from the base and infinite-size grid at WT and FLT Re, $M=1.6$,

$$
\alpha=\mathbf{8}^{\circ} \text {. }
$$

The bar graph in Fig. 13 presents the computed results at the FLT Re along with those computed at the WT $R e$ that were shown earlier. In general, the results in the longitudinal aerodynamic characteristics and the center of pressure over the examined flow conditions and roll angles indicated that the maximum error estimate from the BG to an IG were less than $10 \%$, with an exception. This exception is associated with the computed $C_{N}$ at $M$ $=0.9$ and FLT $R e$ that results in a maximum change of about $18 \%$ due to the grid refinement across the roll angles.

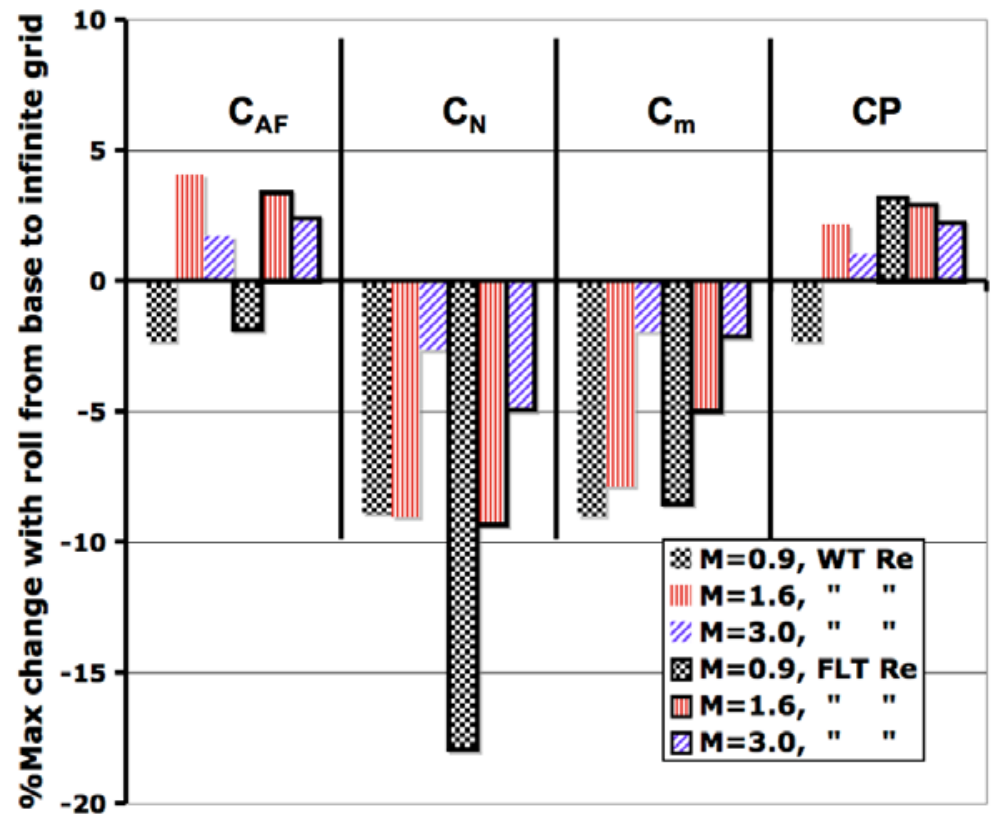

Figure 13. Percent maximum error estimate with roll angle in the computed longitudinal aerodynamic characteristics and the center of pressure from the base to an infinite-size grid for various Mach numbers at $\alpha=8^{\circ}$, WT and FLT Re. 


\section{Concluding Remarks}

Numerical predictions for the longitudinal aerodynamic characteristics of the Ares I class of vehicles, based on the Reynolds averaged Navier-Stokes equations, are presented at flow conditions pertinent to the ascent phase of the flight trajectory. The results were obtained from the NASA Langley developed unstructured grid flow solver, USM3D, and were based on a fully turbulent flow assumption over the entire vehicle surfaces. Due to limited availability of the computer resources, the present analyses were performed only at the selected flow conditions of $\alpha=8^{\circ}, M=0.9,1.6,3.0$ at both flight and wind tunnel Reynolds number. The focus of the analysis is to quantify the error estimates due to iterative convergence grid refinement in the computed aerodynamic coefficients of interest for the vehicle's last design cycle, designated as the A106 configuration. The validity of the approach to compute the associated error estimates, derived from a base grid to an extrapolated infinite-size grid, was first demonstrated on a sub-scaled wind tunnel model at representative flow conditions for which the experimental data existed. Such analysis at the transonic flow conditions revealed a maximum deviation of about $23 \%$ between the computed longitudinal aerodynamic coefficients with the base grid and the measured data across all the roll angles. This maximum deviation from the wind tunnel data was associated with the computed normal force coefficient at the transonic flow condition and was reduced to approximately $16 \%$ based on the infinite-size grid. However, all the computed aerodynamic coefficients with the base grid at the supersonic flow conditions showed a maximum deviation of only about $8 \%$ with that level being improved to approximately $5 \%$ for the infinite-size grid. A maximum error estimate of less than $10 \%$ was found, in the overall computed longitudinal aerodynamic characteristics at wind tunnel Reynolds numbers, between the base and the infinite-size grid across the examined roll angles and Mach numbers. In general, error estimate remained similar for the computations at flight Reynolds number with the exception of the computed normal force coefficient that was increased to approximately $18 \%$ at the transonic Mach number.

\section{Acknowledgement}

The authors would like to thank Edward B. Parlette of ViGYAN, Inc. for generating the unstructured grids used in the present computational analysis.

\section{References}

1. NASA’s Exploration Systems Architecture Study, NASA-TM-2005-214062, Nov. 2005.

2. Huebner, L. D., Smith, R. M., Campbell, J. R., and Taylor, T. L., “Ares I-X Flight Vehicle Similitude to the Ares I Crew Launch Vehicle,” IAC-08-D2.6.7, 59 ${ }^{\text {th }}$ International Astronautical Congress, Sept. 29-Oct. ${ }^{\text {rd }}$ 2008, Glasgow United Kingdom.

3. Frink, N. T., Pirzadeh, S. Z., Pandya, M. J., Bhat M. K. “The NASA Tetrahedral Unstructured Software System,” The Aeronautical Journal, Vol. 104, No. 1040, October 2000, pp. 491-499.

4. Alexandrov, N. M., Atkins, H. L., Bibb, K. L., Biedron R. T., Carpenter, M. H., Gnoffo, P. A., Hammond, D. P., Jones, W. T., Kleb, W. L., Lee-Rausch, E. M., Nielsen, E. J., Park, M. A., Raman, V. V., Roberts, T. W., Thomas, J. L., Vatsa, V. N., Viken, S. A., White, J. A., Wood, W. A., "Team Software Development for Aerothermodynamic and Aerodynamic Analysis and Design,” NASA TM-2003-212421, November 2003.

5. Buning, P. G., Chan, W. M., Renze, K. J., Sondak, D. L., Chiu, I. T., and Slotnick, J. P., OVERFLOW User's Manual, Version 1.6ab, 26 January 1993, NASA Ames Research Center, Moffett Field, CA, Jan. 1993.

6. Abdol-Hamid, K. S., Ghaffari, F., and Parlette, E. B., "Overview of Ares I CFD Ascent Aerodynamic Data Development and Analysis Based on USM3D,” AIAA Paper No. 2011-0015.

7. Spalart, P. R., and Allmaras, S. R., A One-Equation Turbulence Model for Aerodynamic Flows. AIAA Paper No. 1992-0439.

8. Jones, W. P. and Launder, B. E., “The Prediction of Laminarization With a Two-Equation Model of Turbulence,” Int. J. Heat \& Mass Transf., Vol. 15, No. 2, Feb. 1972, pp. 301-314.

9. Menter, F. R., “Improved Two-Equation k-omega Turbulence Models for Aerodynamic Flows,” NASA TM-103975, October 1992.

10. Samareh, J. A., "GridTool: A Surface Modeling and Grid Generation Tool, Proceedings of the Workshop on Surface Modeling, Grid Generation, and Related Issues in CFD Solutions,” NASA Lewis Research Center, Cleveland, OH, NASA CP-3291, 1995, May 9-11, 1995.

11. Pirzadeh, S. Z., “Advanced Unstructured Grid Generation for Complex Aerodynamics Applications,” AIAA Paper No. 2008-7178, August 2008.

12. http://aaac.larc.nasa.gov/tsab/cfdlarc/aiaa-dpw/: 4th AIAA CFD Drag Prediction Workshop (DPW) [cited 28 August 2009].

13. http://hiliftpw.larc.nasa.gov/: “1 $1^{\text {st }}$ AIAA CFD High Lift Prediction Workshop (HiLiftPW).” [cited 28 April 2010 ]. 
14. Cavallo, P. and Grismer, M., “A Parallel Adaptation Package For Three-Dimensional Mixed-Element Unstructured Meshes,” Journal of Aerospace Computing, Information, and Communication 2005, 1542-9423 Vol. 2, No. 11, (433451) doi: 10.2514/1.16220.

15. Erickson, G. E. and Wilcox, F. J., “Ares I Aerodynamic Testing at the NASA Langley Unitary Plan Wind Tunnel," AIAA Paper No. 2011-0999.

16. Pinier, J. T., Niskey, C. J., Hanke, J. L., and Tomek, W. G., “Ares I Aerodynamic Testing at the Boeing Polysonic Wind Tunnel,” AIAA Paper No. 2011-0998. 